FERMILAB-TM-2088

\author{
Jet Calorimetric Trigger
}

Dan Green

Fermi National Accelerator Laboratory

P.O. Box 500, Batavia, Illinois 60510

September 1999 


\section{Disclaimer}

This report was prepared as an account of work sponsored by an agency of the United States Government. Neither the United States Government nor any agency thereof, nor any of their employees, makes any warranty, expressed or implied, or assumes any legal liability or responsibility for the accuracy, completeness, or usefulness of any information, apparatus, product, or process disclosed, or represents that its use would not infringe privately owned rights. Reference herein to any specific commercial product, process, or service by trade name, trademark, manufacturer, or otherwise, does not necessarily constitute or imply its endorsement, recommendation, or favoring by the United States Government or any agency thereof. The views and opinions of authors expressed herein do not necessarily state or reflect those of the United States Government or any agency thereof.

\section{Distribution}

Approved for public release; further dissemination unlimited.

\section{Copyright Notification}

This manuscript has been authored by Universities Research Association, Inc. under contract No. DE-AC02-76CH03000 with the U.S. Department of Energy. The United States Government and the publisher, by accepting the article for publication, acknowledges that the United States Government retains a nonexclusive, paid-up, irrevocable, worldwide license to publish or reproduce the published form of this manuscript, or allow others to do so, for United States Government Purposes. 
Fermilab-TM-2088

\title{
Jet Calorimetric Trigger
}

\author{
Dan Green
}

Fermilab

July, 1999 


\section{Introduction}

The problem in triggering on jets in hadron colliders is that the largest background in the trigger is also a jet. Therefore, we must strive to trigger as incisively as possible. Since the basic physics resides in the transverse momentum, Pt, of the jet, this means to trigger with as sharp a Pt discriminant as possible. The basic problem is different for electrons or photons, where the backgrounds are typically jets. For heavy quarks, like b, the backgrounds are mismeasured secondary vertices. The situation is similar to that for muons, where the largest background is due to real muons from pion decays in particle cascades or from heavy flavor semi-leptonic decays arising from gluon splitting in ordinary QCD jets. As with jets, the issue for the muon trigger is to make as sharp a Pt cut as possible since the background muons dominate at low Pt.

\section{Simple Jet Model}

A simple model of a jet was first constructed. Radiation of gluons from the primary parton was ignored. It is likely that jets which radiate will be "demoted" sufficiently in Pt that they will be lost from the trigger Pt cut. Jets were begun with a transverse momentum Pt. Jet fragments were chosen from a fragmentation function $\mathrm{D}(\mathrm{z})$, where $\mathrm{z}$ is the fraction of the parent longitudinal momentum taken off by the fragment, and with a transverse momentum with respect to the jet axis of $\mathrm{k}_{\mathrm{t}}$.

$$
\begin{aligned}
& \mathrm{zD}(\mathrm{z})=\mathrm{a}(1-\mathrm{z})^{\mathrm{b}}, \mathrm{b}=5 \\
& \mathrm{z}_{\min }=\mathrm{m}_{\pi} / \mathrm{Pt} \\
& \sum \mathrm{z}=1 \\
& \mathrm{~d} \sigma / \mathrm{dk}_{\mathrm{t}}^{2}=\exp \left(-\mathrm{dk}_{\mathrm{t}}^{2}\right)
\end{aligned}
$$

For a $\mathrm{Pt}=100 \mathrm{GeV}$ jet, the mean transverse momentum of a fragment is defined by choosing the parameter $\mathrm{d}$ such that $\left\langle\mathrm{k}_{\mathrm{t}}\right\rangle=0.72 \mathrm{GeV}$. The mean $\mathrm{z}$ was, $\langle\mathrm{z}\rangle=0.04$. The "leading fragment" takes off, on average, about a quarter of the jet energy, $\left\langle\mathrm{z}_{1}\right\rangle=0.25$.

The r.m.s. in pseudorapidity of the jet fragments averaged to 0.45 . Note that no hadronic cascade in the calorimeter was simulated. This approximation is roughly justified because typical calorimetry might occur at a radius $>2.0 \mathrm{~m}$ from the primary vertex. As we will see, we are considering a trigger tower of full width, $\mathrm{d} \eta=0.4$. Therefore, the size of the tower is $\sim 80 \mathrm{x} 80 \mathrm{~cm}$ at large angles. Compared to a typical transverse hadron shower size of $\sim \lambda \sim 15 \mathrm{~cm}$ in $\mathrm{Fe}$ or $\mathrm{Cu}$, we can see that hadron cascade effects are expected to be small.

The transverse energy flow in this model is shown in Fig.1. 


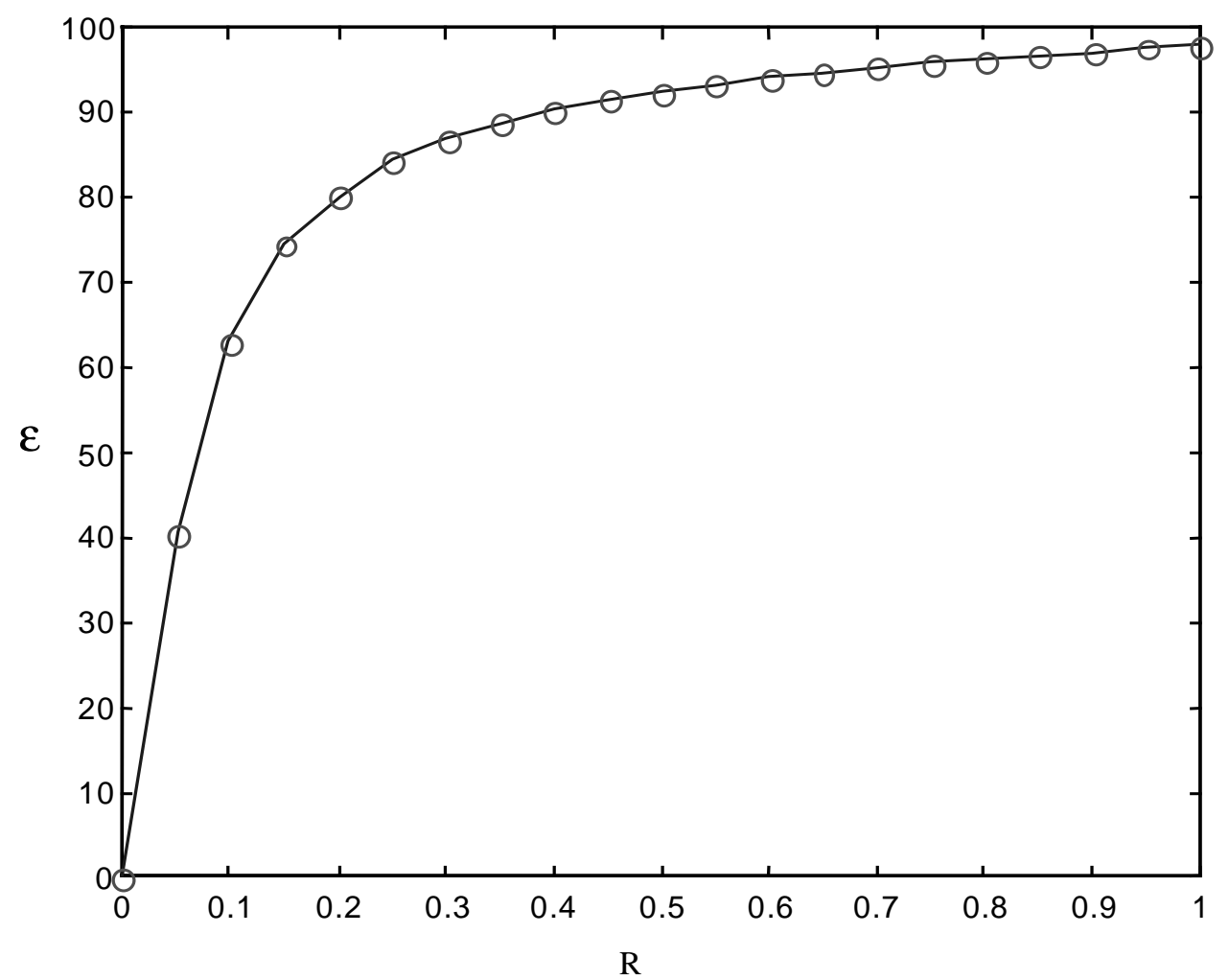

Figure 1: Fraction of the jet energy contained in a cone of radius $\mathrm{R}$ for a $100 \mathrm{GeV}$ jet, where $R=\sqrt{ } \mathrm{d} \eta^{2}+\mathrm{d} \varphi^{2}$.

Note that $50 \%$ of the jet energy is contained in a "core" of cone radius $<0.075$. This core is due to the leading particle in the jet taking off $\sim 1 / 4$ of the energy on average. With a mean $\mathrm{kt}$ of $\sim 0.72$ and a mean $\mathrm{z}$ of $\sim 0.25$, the leading fragment is emitted with $\mathrm{R} \sim 0.029$. this prediction is consistent with the behavior displayed in Fig.1. There is also a long tail, where $80 \%$ of the jet energy is contained in a cone radius of $\mathrm{R}<0.2$. Existing D0 data ${ }^{[1]}$ for $\mathrm{Pt}=53 \mathrm{GeV}$ jets is somewhat broader in $\mathrm{R}$. The conclusions of this note are insensitive to the fine details of the jet $\mathrm{R}$ distribution; the main effects are due to the geometry of the trigger towers themselves.

\section{Trigger Towers}

Consider a calorimeter with physical tower size of $d \eta=0.1$. Suppose these towers are summed into trigger towers with size $d \eta=0.4(\mathrm{R} \sim .0 .2)$ formed by summing 16 of the physical towers. If the calorimetry covers a pseudorapidity down to $\eta=5$, then there are $16 \times 25=400$ trigger towers in $\eta \times \varphi$. There are thus 400 trigger elements, a not intractably large number.

It is fairly standard practice in offline jet algorithms to define a jet using a seed tower surrounded by a cone of size $\mathrm{R}=0.7$. If we adopt a $3 \times 3$ set of trigger towers with a full width of $\mathrm{d} \eta=1.2$, then the radius of the $3 \times 3$ array for a jet which strikes the center of 
the central trigger tower is $\mathrm{R} \sim 0.6$. As seen in Fig.1, in that case, $\sim 90 \%$ of the jet energy is deposited, on average, in the $3 \times 3$ array.

A simple Monte Carlo model was written where jets were set to uniformly illuminate the central trigger tower of the $3 \times 3$ array. For $\mathrm{Pt}=100 \mathrm{GeV}$ jets, the resultant energy deposition for a few single jets is shown in Fig.2.
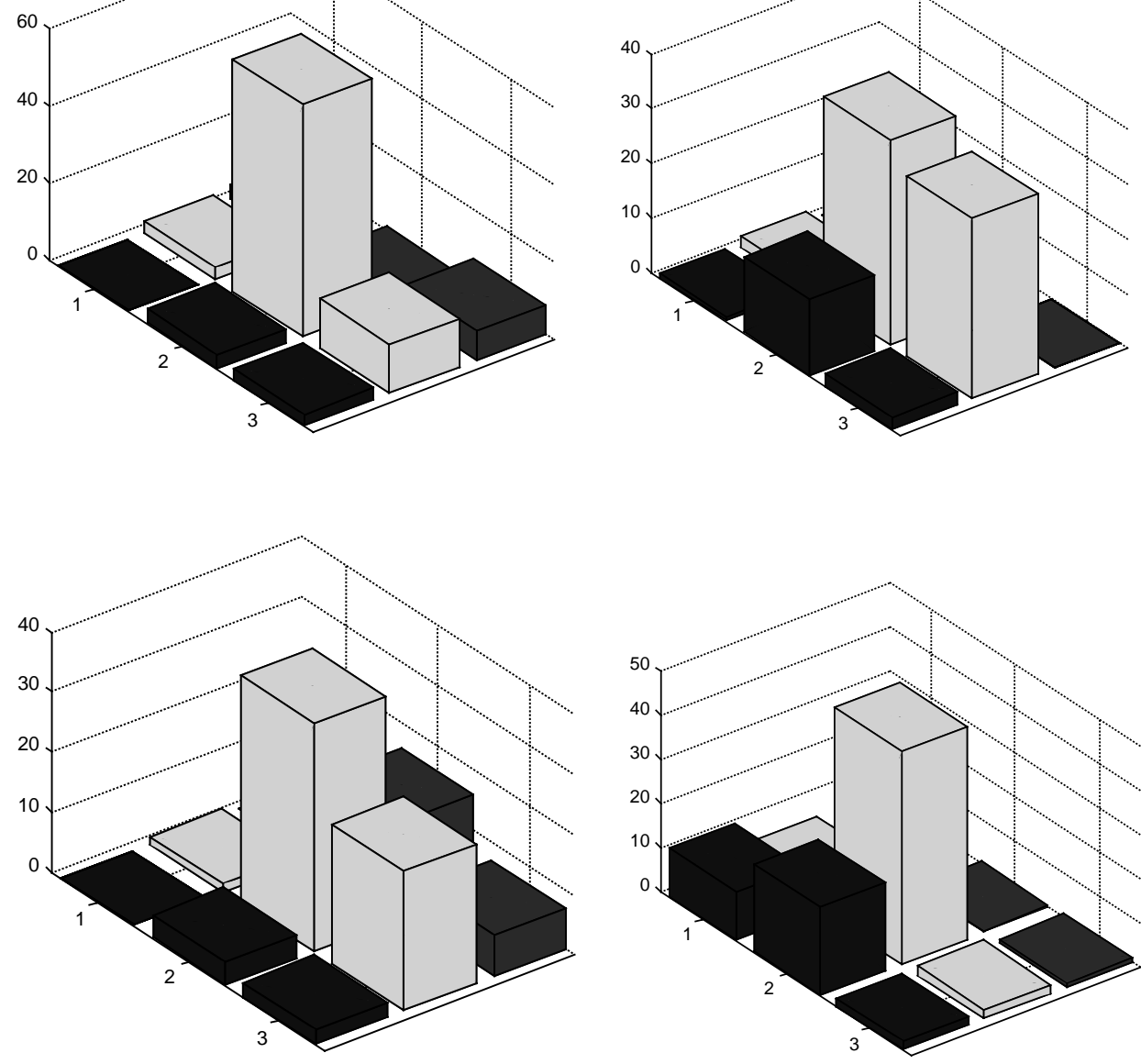

$\mathrm{E}(\mathrm{GeV})$

Figure 2: Energy deposition in the 3 x 3 trigger tower array for $\mathrm{Pt}=100 \mathrm{GeV}$ jets.

Note that sometimes most of the jet energy goes into the central tower of the array Fig.2a. From Fig.1 we expect $\sim 80 \%$ of the energy to go into the central tower in this case. However, it is inevitable that the jet axis sometimes impacts an edge of the central tower. In that case, we expect only $\sim 1 / 2$ the energy in the central tower, Fig. $2 b$. The worst case is an impact at the corner of 4 trigger towers. In that case we expect $\sim 1 / 4$ of the energy in 4 trigger towers. Therefore, the energy deposited in the seed tower is not 
expected to be a reliable measure of the true jet energy due to simple geometric limitations.

The histograms of the energy in the central tower for $\mathrm{Pt}=100 \mathrm{GeV}$ jets and in the $3 \mathrm{x} 3$ array are shown in Fig. 3 and Fig. 4 respectively. Note that there is, as yet, no energy smearing due to the energy resolution of the calorimeter employed to measure hadronic energy.

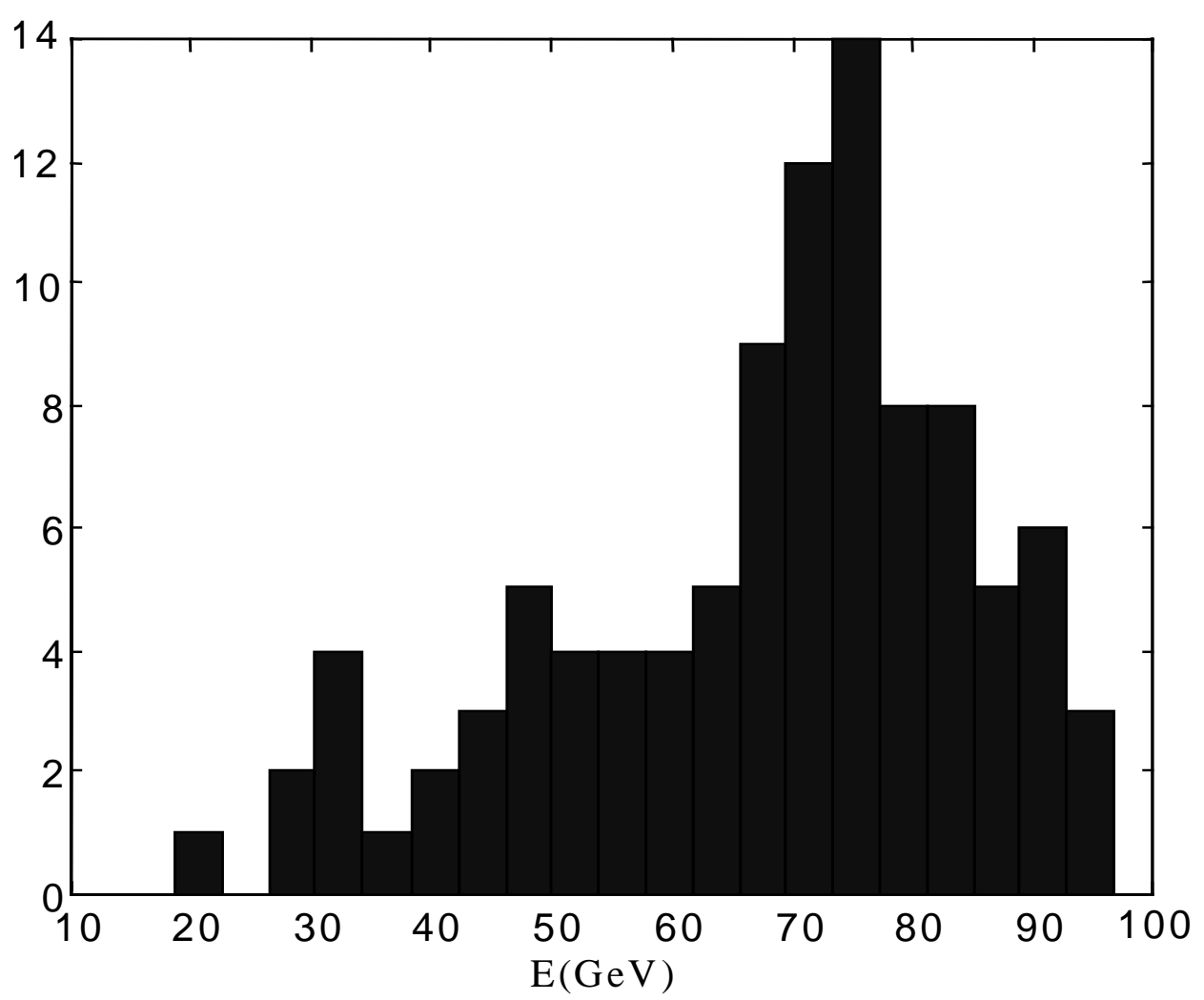

Figure 3: Energy deposited in the central tower of a $3 \times 3$ trigger tower array for a $\mathrm{Pt}=$ $100 \mathrm{GeV}$ jet. 


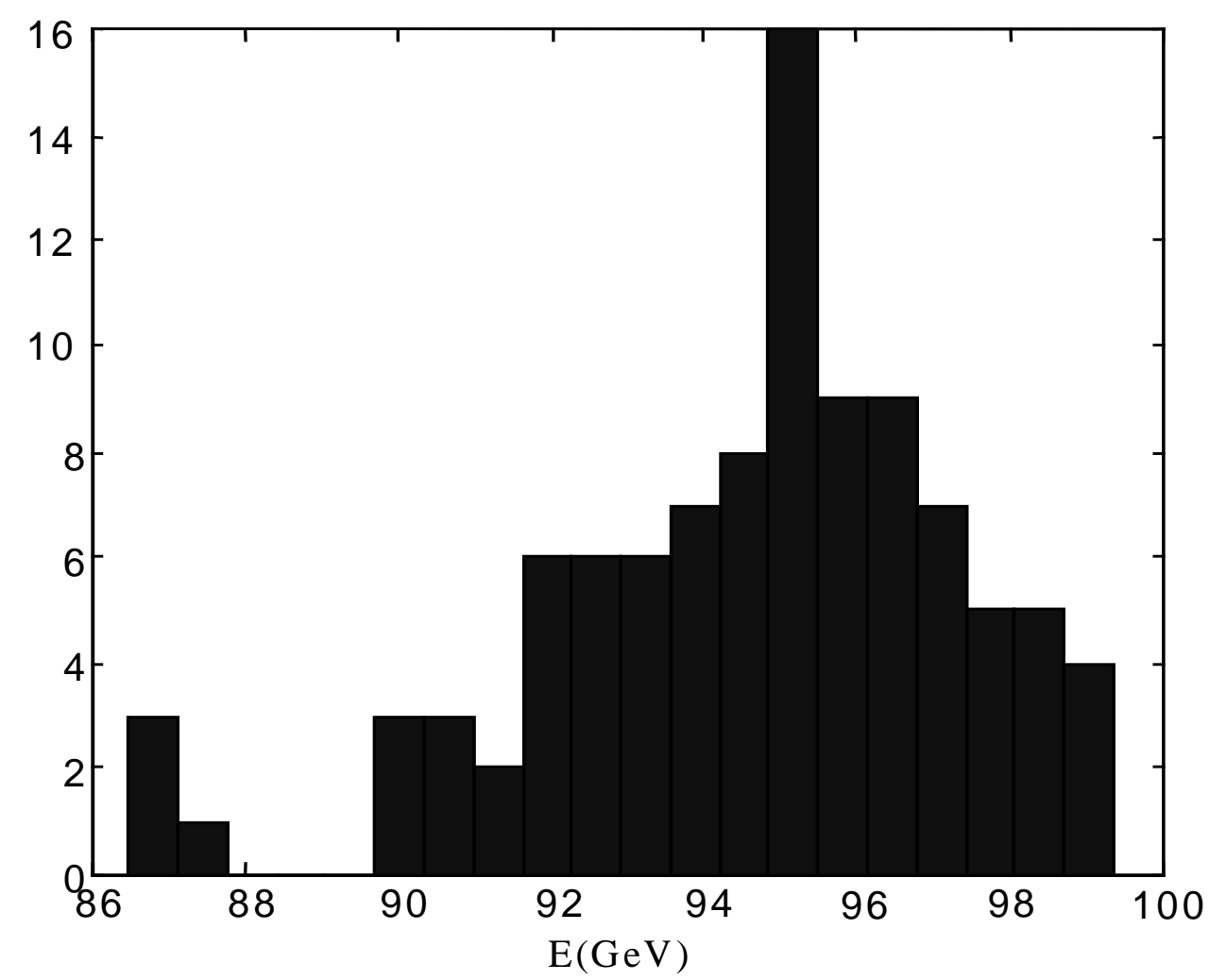

Figure 4: Energy deposited in a 3 x 3 trigger tower array for a $\mathrm{Pt}=100 \mathrm{GeV}$ jet.

As expected from geometry, the energy in the central tower can be only $\sim 1 / 4$ of the jet energy. Thus, efficient detection would require a threshold of $\sim 30 \mathrm{GeV}$ for a $\mathrm{Pt}=100$ $\mathrm{GeV}$ jet. As regards the full $3 \times 3$ array, we can ask for $90 \%$ of the jet energy because in this case, $\mathrm{R} \sim 0.6$, regardless of the impact point in the central tower. From Fig.1, we expect $90 \%$ jet energy containment in this case. The scatter plot of the central tower and the $3 \times 3$ array energies is shown in Fig.5.

Note that the efficiency for a cut $\mathrm{E} 1>30 \mathrm{GeV}$ and $\mathrm{E} 2>90 \mathrm{GeV}$, where $\mathrm{E} 1$ is the energy in the central tower and E2 is the energy observed in the $3 \times 3$ tower array, is $93 \%$ for a $100 \mathrm{GeV}$ jet. Note that there is not a high degree of correlation between the two energies, as seen in Fig.5. Thus, a sharp edge will require both requirements to be imposed. We cannot expect the central tower to be well correlated with the true jet energy, as mentioned above.

Note that with 400 trigger towers there are 44 full $3 \times 3$ arrays. Thus, we can identify 44 distinct jets in an event, in principle, if we require that found jets be entirely distinct. In practice, no more than 4 jets are typically contemplated as a potential trigger requirement by either ATLAS ${ }^{[2]}$ or CMS ${ }^{[3]}$. Note also that if a jet is defined by a threshold on a single tower of E1 and a second cut on a $3 \times 3$ array centered on that single tower of E2, then we must be careful about double counting a single jet. As can be seen looking at Fig.2b and Fig.2c, two jets would be reported in this case. It is necessary that in a case 
where there are 2 adjacent central towers reporting jets, that only a single jet be reported, choosing the one with the largest $3 \times 3$ total energy. As noted above, this additional cut places no serious restriction on the counting of jets for triggers on multiple jets. In fact, the procedure should yield an accurate count of the number of jets in the event.

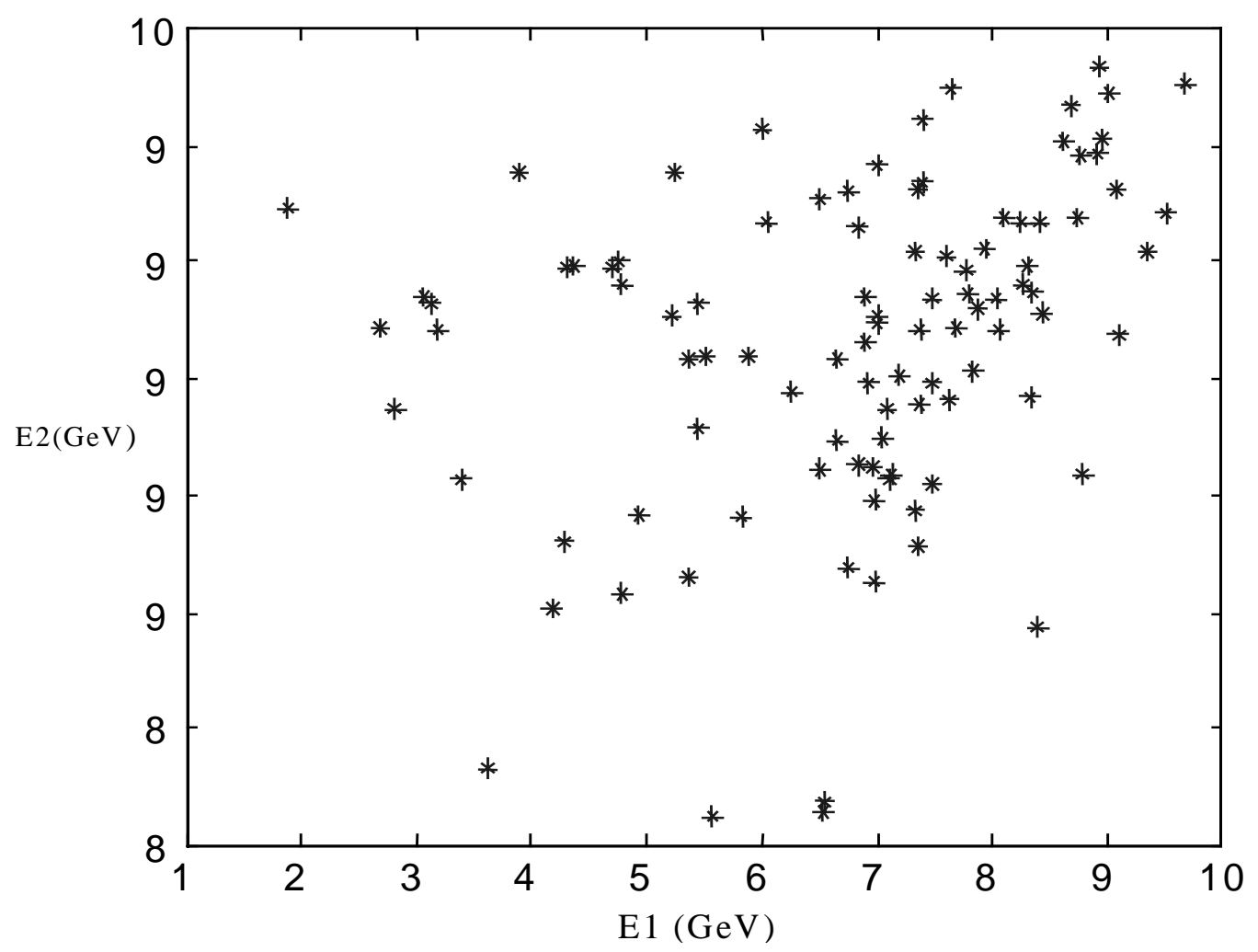

Figure 5: Energy in the central trigger tower vs. the energy in the full $3 \times 3$ trigger tower array for $100 \mathrm{Pt}=100 \mathrm{GeV}$ jets.

\section{Efficiencies}

The response of a single central tower and a central tower and the associated $3 \times 3$ array to jets of different Pt is shown in Fig. 6 and Fig.7. There were 200 jets generated per point, so $100 \%$ efficiency finds 200 jets. The threshold energies for Fig. 6 are E1 $=30$ $\mathrm{GeV}$ and $\mathrm{E} 2=90 \mathrm{GeV}$. These values are appropriate to 50 and $100 \mathrm{GeV}$ jet thresholds respectively for single trigger tower and single and $3 \times 3$ array. For Fig.7 the thresholds are $\mathrm{E} 1=15 \mathrm{GeV}$ and $\mathrm{E} 2=45 \mathrm{GeV}$. The $50 \%$ efficiency in this case is $\sim 30 \mathrm{GeV}$ for a single tower, and $50 \mathrm{GeV}$ for the single and $3 \times 3$ array trigger. 


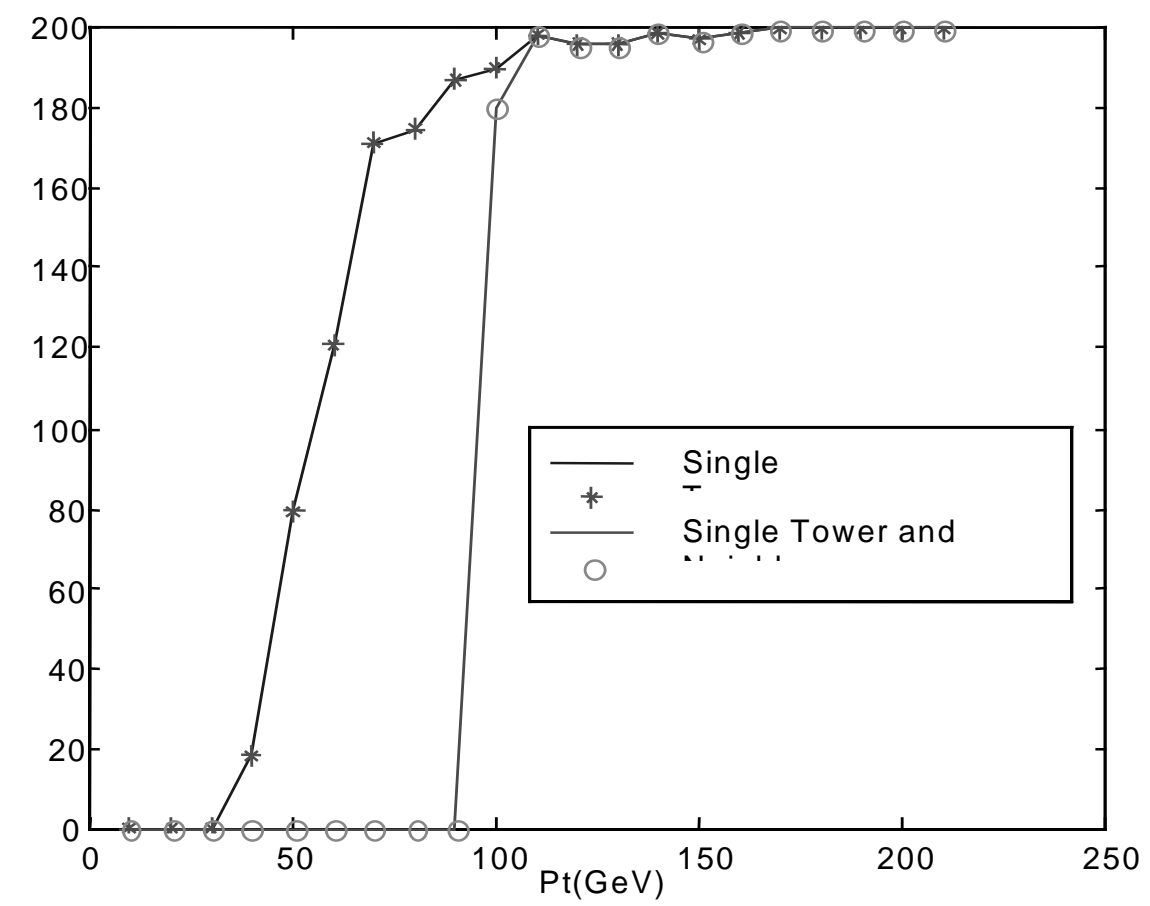

Figure 6: Jet efficiency, jet events which pass for 200 generated, for single tower and single tower and $3 \times 3$ array as a function of jet $\mathrm{Pt}$ for $\mathrm{E} 1>30 \mathrm{GeV}$ and $\mathrm{E} 2>90 \mathrm{GeV}$.

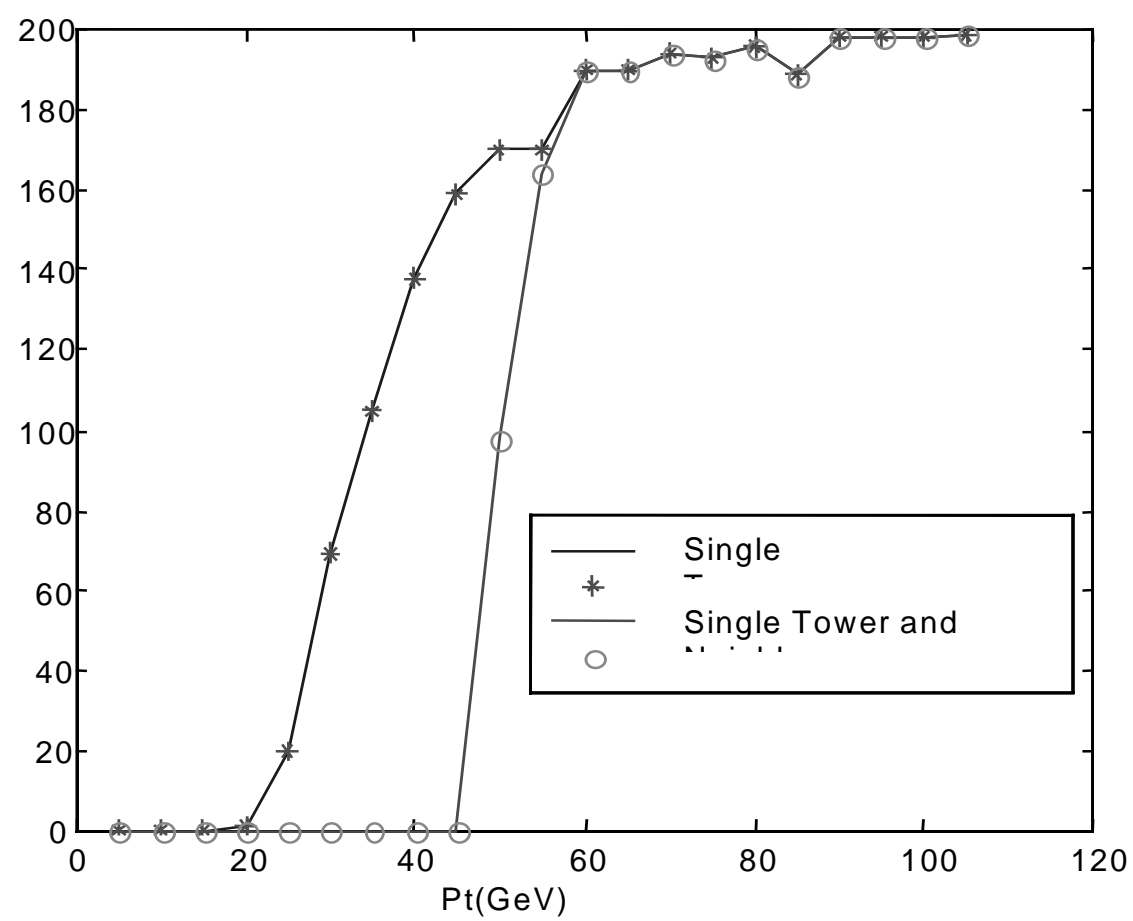

Figure 7: Jet efficiency, jet events which pass for 200 generated, for single tower and single tower and $3 \times 3$ array as a function of jet $\mathrm{Pt}$ for $\mathrm{E} 1>15 \mathrm{GeV}$ and $\mathrm{E} 2>45 \mathrm{GeV}$. 
Comparing Fig. 6 and Fig. 7, - single tower from Fig.6 and single tower + neighbors from Fig. 7 - it is clear that the latter algorithm gives a rather sharper turn on curve for jets. It is also clear that the "AND" of the single tower with energy above E1 and the $3 \times 3$ array above E2 yields a very sharp turn on efficiency curve.

It might be argued that the energy resolution of the calorimeter will wash out these conclusions. In order to study this effect, the jet fragments were smeared in energy by dz $=100 \% \sqrt{\mathrm{z}}$. This is a crude representation of the CMS calorimeter response ${ }^{[4]}$.The results are given in Fig.8 and Fig.9. A set of threshold appropriate to rather low energy jets, E1 = $15 \mathrm{GeV}$ and $\mathrm{E} 2=45 \mathrm{GeV}$ was chosen.

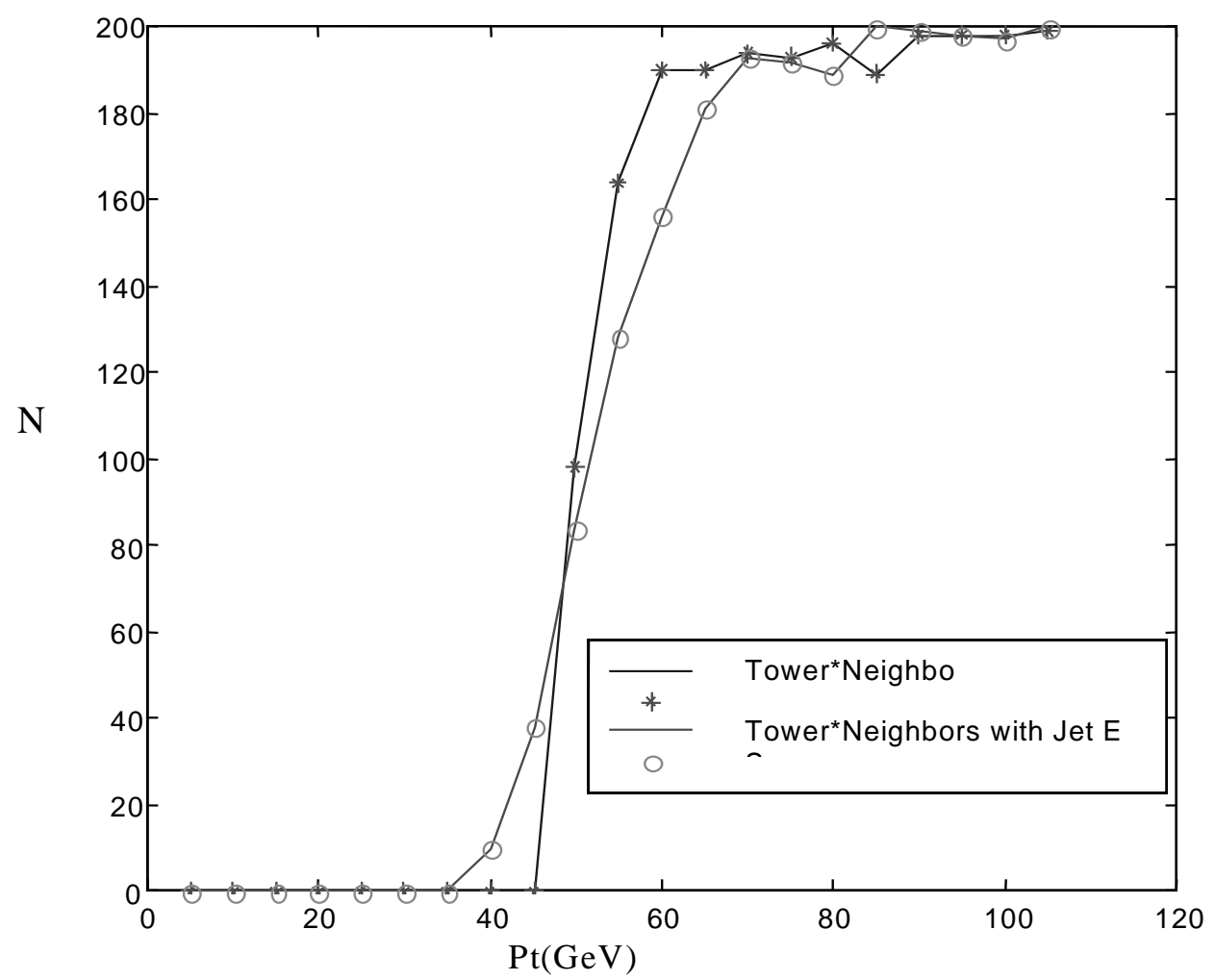

Figure 8: Number of accepted jets out of 200 trials as a function of jet Pt. The single tower AND $3 \times 3$ array trigger algorithm was used for perfectly measured jets and jets with energy errors due to calorimetry. 


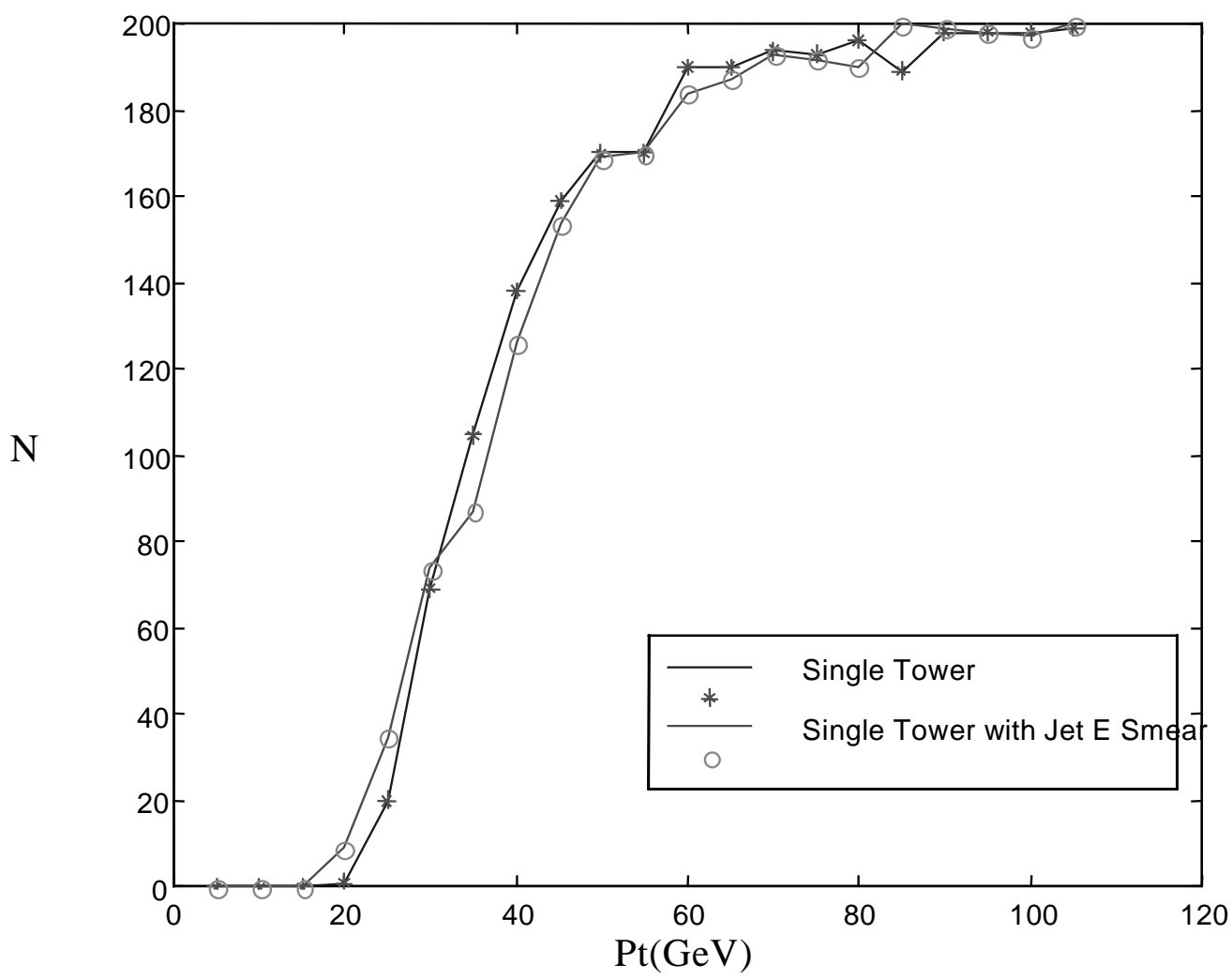

Figure 9: Number of accepted jets out of 200 trials as a function of jet Pt. The single tower trigger algorithm was used for perfectly measured jets and jets with energy errors due to calorimetry.

Note in Fig.8 that there is a noticeable degradation in trigger turn on sharpness due to jet energy measurements. This means that the jet measurement dominates the trigger turn on curve. This is in accord with the "first do no harm" school of triggering. Design a trigger such that it in itself does not contribute to the sharpness of the jet definition. As stated in the beginning, since the background to jets is jets, the sharpness is all. In contrast, the effect of energy mismeasure is dominated by the trigger itself for the single tower trigger, as seen in Fig.9.

Note that, at low Pt the jet cross section, on dimensional grounds, goes as, $\mathrm{d} \sigma / \mathrm{dPt} \sim 1 / \mathrm{Pt}^{3}$. Hence a factor of 2 in $\mathrm{Pt}$ is a factor of 8 in cross section. Therefore, although the turn on curve in Fig.9 may appear innocuous, the rate with respect to that from Fig. 8 is much larger ${ }^{[5]}$. For example, the single jet tower with a $15 \mathrm{GeV}$ threshold is $\sim 100 \%$ efficient for $\mathrm{Pt}>60 \mathrm{GeV}$. It is also $50 \%$ efficient for $40 \mathrm{GeV}$ and $20 \%$ efficient for $25 \mathrm{GeV}$. The rate for $40 \mathrm{GeV}$ jets leaking in is 1.7 times that for $60 \mathrm{GeV}$ jets. The rate for $25 \mathrm{GeV}$ jets leaking in is 2.8 times that of $60 \mathrm{GeV}$ jets. Therefore, to get 1 fully efficient $60 \mathrm{GeV}$ jet we must take several times more lower energy jets which leak through the single tower trigger. This effect is illustrated in Fig. 10 and Fig. 11. 


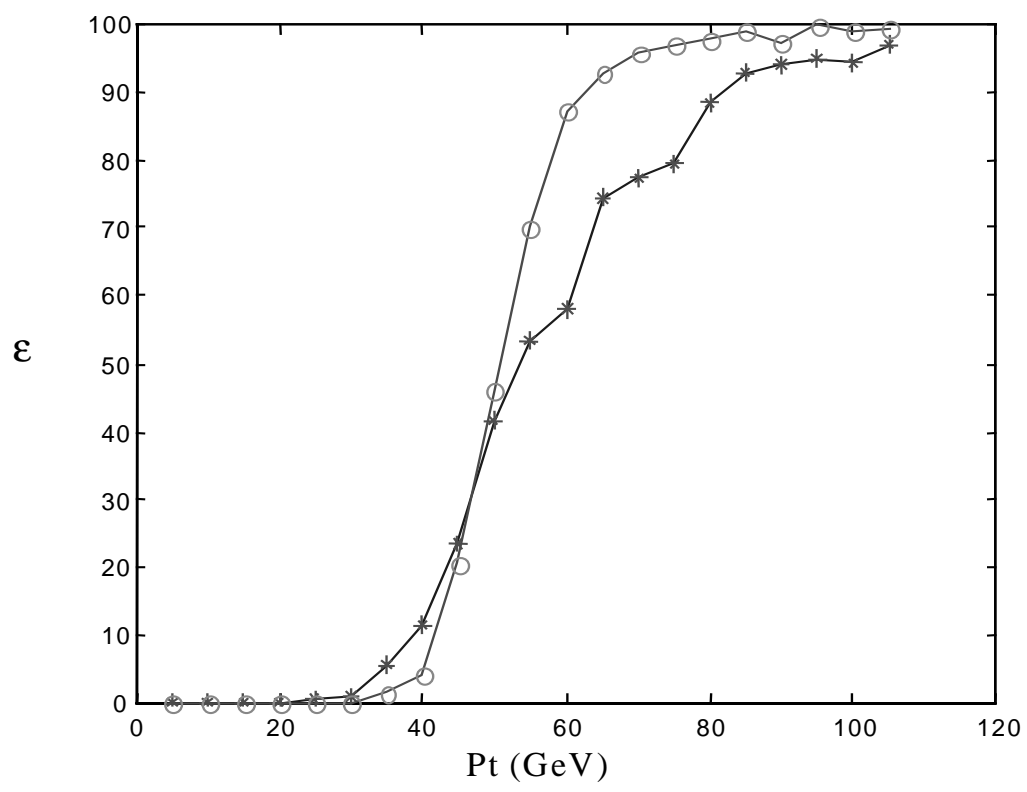

Figure 10: Trigger efficiency for single tower and tower + neighbors with threshold energies chosen to give $50 \%$ efficiency at $\sim 50 \mathrm{GeV}$. Jet energies are smeared by the expected calorimetric resolution.

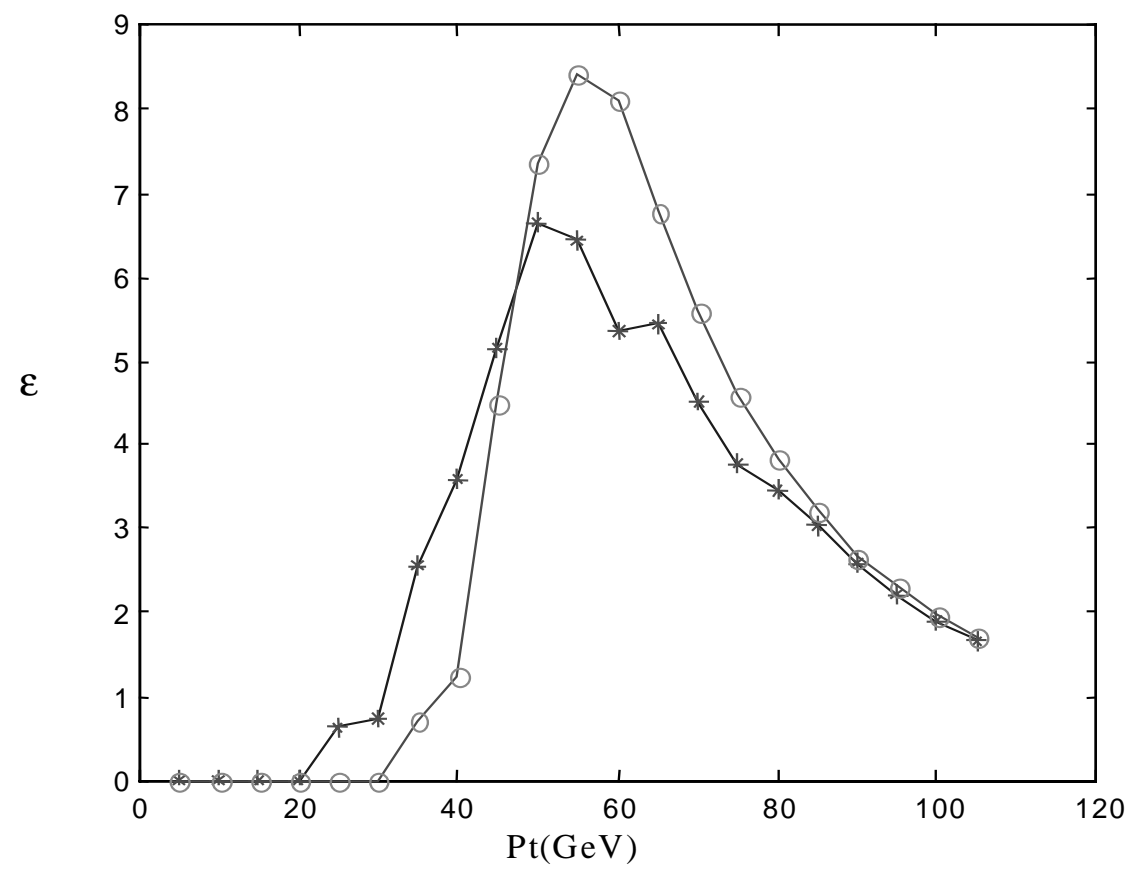

Figure 11: Trigger rates as a function of jet Pt using the efficiencies given in Fig.10 and assuming a $1 / \mathrm{Pt}^{3}$ jet dependence.

Note also that the exact details of the turn on curve are due to the details of the jet fragmentation process as well as the geometric effects we focus on here. This is surely 
different for gluon, light quark, and heavy quark jets. Therefore, it is extremely unlikely that the events in the Pt range where the efficiency is $<1$ will ever be used in a reliable physics analysis ${ }^{[5]}$. Nevertheless, these events dominate the trigger bandwidth, thus precluding other triggers from usefully employing that bandwidth. Therefore, it is incumbent upon us to design a trigger which, indeed, does not in itself define the response of an experiment, but rather the unavoidable intrinsic resolution of the calorimetry.

\section{References}

1. Transverse Energy Distributions within Jets in pp Collisions at $\sqrt{s}_{\mathrm{s}}=1.8 \mathrm{TeV}$, Phys. Lett., pg. 500 B357 (1995)

2. ATLAS - L1 Trigger, Technical Design report, June 24, 1998

3. S. Dasu et al., CMS TN/95-11, August 20, 1995

4. CMS - HCAL, Technical Design Report, June, 1997

5. J. Womersley, private communication. A factor $10 \mathrm{in} \mathrm{L1} \mathrm{rate} \mathrm{reduction} \mathrm{is} \mathrm{modeled} \mathrm{in} \mathrm{D0} \mathrm{for} \mathrm{Run} \mathrm{I}$ D0 have never used low Pt jets in a refereed publication due to acceptance uncertainties. 